\title{
¿PARA QUÉ SIRVE LA HISTORIA DE LA FILOSOFÍA? A PROPÓSITO DE LA TEORÍA DE LA PERCEPCIÓN DE JOHN R. SEARLE
}

\author{
JOSÉ ANTONIO VALDIVIA FUENZALIDA \\ Universidad Adolfo Ibáñez, Chile
}

\begin{abstract}
RESUMEN: John Searle defiende el «realismo directo», el cual sostiene que el objeto directo de la percepción es la realidad extra-mental. En conformidad con esta teoría, no hay ningún tipo de entidad intermediaria entre el acto perceptivo y la cosa percibida, como una «representación», "semejanza» o «especie». De hecho, Searle desarrolla sistemáticamente una teoría de la percepción que despliega las posibilidades explicativas de la tesis mencionada. En el presente artículo, se comentan ciertos aspectos de esta con el propósito de compararlos con teorías del conocimiento propuestas por pensadores medievales. Se intenta poner de manifiesto que la teoría de Searle no ofrece nada radicalmente nuevo y que cae en ciertos errores que pudo evitar de haber conocido los trabajos de la historiografía medieval que se ocupan de estos temas. Así, se reivindica la importancia de la historia de la filosofía.

PALABRAS CLAVES: Realismo; representacionalismo; intencionalidad; Pedro de Olivi; Tomás de Aquino; escepticismo
\end{abstract}

\section{What is the Purpose of History of Philosophy? Regarding John R. Searle's Theory of Perception}

ABSTRACT: John Searle defends "Direct Realism», which argues that the direct object of perception is the extra-mental reality. According to this theory, there is no intermediary entity between the act of perception and the perceived thing, as a «representation», «resemblance», or "species». Actually, Searle systematically develops a theory of perception which displays the explanatory possibilities of the mentioned thesis. In the present article, we discussed some aspects of the mentioned thesis with the purpose of comparing them with selective medieval thinkers cognitive theories. We intend to show that Searle's theory offers nothing radically new, and that he falls into avoidable mistakes if he had had a better understanding of medieval historiography that cover such issues. Thus, we intend to assert the importance of the History of Philosophy.

KEY WORDS: Realism; Representationalism; Intentionality; Peter of Olivi; Thomas Aquinas; Skepticism

\section{INTRODUCCIÓN}

¿Por qué seguir leyendo a los filósofos del pasado? ¿Qué sentido tiene estudiarlos en profundidad y escribir sobre ellos? Rémi Brague, uno de los más reconocidos historiadores actuales de la filosofía medieval, a propósito de la originalidad que debía atribuirse a Averroes con respecto a Aristóteles, se expresaba en estos términos:

«(..) aquellos que buscan la originalidad a cualquier precio y pretenden, con bombos y platillos, destruir hasta la raíz todo lo que ha sido realizado antes que ellos, bastante a menudo no hacen más que recalentar y servir, con una salsa insípida, algún vejestorio cuya única novedad era haber sido olvidado. Por el 
contrario, aquellos que no tienen otra ambición consciente o, al menos, ostentada, más que la de tomar parte de una gran tradición para aportar a ella con ciertas adaptaciones mínimas y actualizaciones, se revelan a menudo, ante la mirada retrospectiva, como poderosos innovadores, incluso como revolucionarios» ${ }^{1}$.

En el presente trabajo, pretendo mostrar que, comparada con la teoría medieval de la percepción, aquella que ha sido propuesta recientemente por John Searle en su último libro ${ }^{2}$ parece, en los términos de Brague, «un plato ya conocido y recalentado». Con el propósito de superar las deficiencias que poseería la epistemología desde Descartes, Searle propone que adoptemos una nueva perspectiva que tomaría como punto de partida la siguiente premisa: el mundo extra-mental existe y la percepción sensible nos lo presenta tal como es en sí mismo. Igualmente, propone que la discusión en torno a la percepción debe suponer que su objeto directo es la realidad extra-mental. Esto le permite ocuparse de una serie de nuevos temas que interesan a cualquiera que desee entender el fenómeno de la intencionalidad del conocimiento y, en particular, de la percepción. Comparada con la tradición epistemológica que se viene desarrollando desde el siglo XVII, esta nueva perspectiva vuelve tal vez original la teoría del filósofo norteamericano. No obstante, para el especialista de la gnoseología medieval, ciertos puntos de la teoría de este autor le parecerán bastante decepcionantes. La razón es que dicho especialista frecuenta sistemáticamente una serie de teorías y argumentos de gran sutileza en torno al tema de la percepción abordado desde la misma perspectiva realista de Searle.

Para poner de manifiesto lo anterior, desarrollaré algunos puntos esenciales de la teoría de Searle y los compararé con algunas teorías medievales, según el modo en que han sido explicadas y comentadas por los historiadores actuales. Mi intención es reivindicar la importancia de que los «filósofos profesionales» estén al tanto de la historia de la filosofía y, en particular, de la filosofía medieval. No solo por el hecho de que esta disciplina nos permita comprender mejor nuestro pasado, sino que por revelarse apta para aportar con ideas susceptibles de ser teóricamente útiles en las discusiones filosóficas actuales. Intentaré mostrar que lo anterior vale especialmente para la filosofía medieval en lo que respecta al tema del conocimiento, aun cuando los historiadores-filósofos de este período son, muchas veces, francamente ignorados por los filósofos contemporáneos

\section{El REALIsmo COMO PUnTO DE PARTida}

No cabe la menor duda de que Searle propone abordar los temas epistemológicos tomando como punto de partida el realismo. No según la acepción que opone este término al nominalismo en relación al famoso problema del estatuto ontológico de los conceptos universales. Más bien, según una acepción mucho más simple y

1 Brague, R., Au moyen du Moyen Âge. Philosophies médiévales en chrétienté, judaïsme et islam, Flammarion, Paris 2008 (reimpresión), p. 404.

2 Searle, J. R., Seeing Things as they are: A Theory of Perception, Oxford University Press, New York, 2015. Cada vez que se haga referencia a esta obra se utilizará la siguiente abreviación: Seeing Things. También utilizaré Intentionality. An essay in the philosophy of mind, Cambridge, Cambridge University Press, 1983, que será abreviada de esta forma: Intentionality. 
que no requiere ni siquiera una toma de posición filosófica consciente. Me refiero al realismo entendido como la aceptación de dos cosas: (1) existe un mundo extramental que no depende en su ser de la percepción que se tenga de él (lo que se opone al idealismo radical); (2) este mundo extra-mental es en sí mismo tal como se presenta a la percepción sensible (lo que se opone, por ejemplo, a lo que algunos llaman "realismo científico», que afirma que existe el mundo extra-mental, pero que este no tiene el aspecto que se presenta ante los sentidos ${ }^{3}$ ). Searle se impone a sí mismo la tarea de elaborar una teoría del conocimiento que explique la percepción adoptando el supuesto mencionado. Este supuesto realista habría sido ignorado por toda la tradición epistemológica iniciada por Descartes. El escepticismo sería uno de los resultados más notables de lo anterior. Pero otro resultado indeseable habría sido, según Searle, el que la epistemología moderna no se habría ocupado de las cuestiones que realmente interesan, por haberse enfocado en dar una respuesta satisfactoria al problema de cómo es posible justificar que conozcamos la realidad con certeza si solo percibimos nuestras representaciones y no la realidad misma ${ }^{4}$.

Profundicemos un poco en este punto de partida adoptado por Searle. Lo primero que debe subrayarse es que admite la noción tradicional de verdad como «adecuación». Una proposición o creencia es verdadera cuando esta expresa lo que es la realidad a la cual se refiere. Pero esto no es una novedad en el contexto de la filosofía de las ciencias contemporáneas ni de la filosofía analítica donde, generalmente, se admite esta noción de verdad ${ }^{5}$. No obstante, bajo este presupuesto, Searle adopta un punto de partida que se puede considerar como relativamente novedoso y problemático, a saber, que la percepción sensible presenta la realidad objetiva tal cual es, lo que comúnmente es denominado "realismo ingenuo» ${ }^{6}$. El realismo científico, en efecto, si bien acepta la noción tradicional de verdad, considera que la percepción sensible se limita a formar en la mente un contenido que no se adapta a lo que las cosas son en sí mismas ${ }^{7}$. Searle, en cambio, se opone abiertamente a esta posición. Más aún, su objetivo principal es la elaboración de una teoría de la percepción sensible que explique este fenómeno bajo el presupuesto de que la realidad es percibida tal cual es. Para él, lo que se nos manifiesta por medio de los sentidos se adapta o ajusta (fit) a lo que las cosas son ${ }^{8}$. Comparándola con la Crítica de la razón pura de Kant, que asume un presupuesto contrario, Searle declara que su propia teoría se presenta como la consecuencia necesaria del «presupuesto» (assumption) realista, es decir, de aquel que admite que la percepción tiene un acceso directo a la realidad tal cual es ${ }^{9}$.

3 Sobre el realismo científico, cf. Torretti, R., Estudios filosóficos (1986-2006), Ediciones UDP, Santiago, 2007, pp. 75-82.

${ }_{4}$ Seeing things, p. 217: «(...) given that all we can perceive are our own sense data, how is it possible to gain secure and certain knowledge of the external world?».

5 Boghossian, P., Fear of Knowledge. Against Relativism and Constructivism, Oxford University Press, Oxford, 2006, pp. 10-20.

$6 \quad$ Seeing things, pp. 15-20; Intentionality., pp. 11-13, 57-61.

7 TorretTi, R., Estudios filosóficos, op.cit., pp. 75-82.

8 Seeing things, pp. 33-37; Intentionality, pp. 42-45.

9 Seeing things, pp. 31, 94-98. Cf. Intentionality., p. 57: «The account of visual perception that I have been arguing for so far is, I guess, a versión of 'naive' (direct, common sense) realism $(\ldots) »$. 
Lo anterior se verifica fundamentalmente en aquello que, para el autor, es el objetivo principal de su libro, a saber, proponer una teoría que explique cómo la experiencia perceptiva —ontológicamente subjetiva y con características propiaspuede ser una "presentación» (presentation) ${ }^{10}$ de propiedades y características del mundo objetivo. La pregunta no es, para Searle, el saber si la experiencia subjetiva presenta o no las características de la realidad objetiva, ni tampoco la de establecer un criterio que nos permita establecer cuándo lo hace y cuándo no. La pregunta es, por el contrario, el saber cómo lo hace, bajo el supuesto permanente de que efectivamente lo hace: «¿Cómo las características específicas del campo visual ontológicamente subjetivo presenta características del campo visual objetivo como sus condiciones de satisfacción [conditions of satisfaction]?» ${ }^{11}$.

Loanteriorse comprende mejor cuandonos referimos a algunas delas características que Searle confiere a la percepción. Según él, esta última es de naturaleza diferente a la realidad percibida, lo cual se verifica en tres puntos: (1) la percepción es «subjetiva» y la realidad «objetiva»; (2) la percepción es «cualitativa», la realidad «material»; (3) la percepción es «intencional», la realidad «física» ${ }^{12}$. Con respecto a esta última idea, Searle indica que, aunque sea el resultado de un proceso causal de orden físico, la percepción no se identifica con este proceso. Aquella es esencialmente intencional, mientras que este último es «no intencional», dado que no está referido a otra $\operatorname{cosa}^{13}$.

En definitiva, ante las consideraciones precedentes, la pregunta que surge es: ¿cómo una experiencia de orden subjetivo e irreductible a lo físico puede ser apta para manifestar la realidad física tal cual es?, ¿cómo una realidad esencialmente diferente a otra puede servir para presentarla fielmente? Se ve cómo, para Searle, la cuestión no es justificar la posibilidad de conocer perceptivamente el mundo extra-mental, sino que encontrar una explicación satisfactoria sobre cómo la experiencia subjetiva permite, a pesar de sus características, dicho conocimiento. Por esto mismo, se vuelve necesario describir el proceso que explica el fenómeno de la manifestación o «presentación» de la realidad en cada experiencia perceptiva, proceso que incluye una serie de elementos causales tanto de orden intencional o inmaterial como de orden físico ${ }^{14}$. En efecto, aun cuando "[h] ay una compleja historia causal de índole no intencional que posibilita el funcionamiento de la intencionalidad $»^{15}$, nada en ese proceso considerado en sí mismo es parte del «contenido intencional» (intentional content) ni constituye el «objeto intencional» (intentional content). En otros términos, si bien la experiencia perceptiva implica todo un proceso causal complejo que envuelve la acción del cerebro y que es

10 Seeing things, pp. 41, 61; Intentionality, p. 46.

11 Seeing things, p. 118: "The question we are addressing is the more specific question, 'How do specific features of the ontologically subjective visual field present features of the objective visual field as their conditions of satisfaction». Cf. Intentionality, pp. 37-45.

12 Seeing things, pp. 14-17, 46-49, 105-110.

13 Ibid., pp. 108-109.

14 Ibid., pp. 108-109, 118-121. Cf. Intentionality, p. 37: «(...) there is a long story about how it works in physical optics and in neurobiology, but that is not what I mean. I mean how does it work conceptually (...)».

15 Seeing things, p. 109: «There is a very complex non-intentional causal story, which enables the intentionality to work». 
suficientemente explicado por la neurobiología, existe un aspecto de la experiencia perceptiva que es irreductible a este último y requiere un tratamiento especial.

Ahora bien, aquí se nos revela el primer aspecto de la obra de Searle que merece ser comparado con la gnoseología medieval. En efecto, ocurre que el punto de partida descrito era asumido de manera completamente natural por los autores de la Edad Media. Cuando estos abordaban problemas relacionados con el conocimiento, no buscaban averiguar si podemos conocer algo, ni buscaban establecer un criterio que permitiera saber cuándo conocemos con certeza y cuándo no. John Haldane, por ejemplo, dice que la razón por la cual los filósofos medievales no se planteaban el problema de la inducción era que su objetivo no era justificar la certeza de nuestras percepciones ni de nuestros conceptos universales sino que, por el contrario, explicar dicha certeza dando por hecho que la podíamos obtener $^{16}$. Para los pensadores de esa época, los sentidos nos daban efectivamente un acceso fiel al mundo, al igual que los conceptos que surgen directamente de estos últimos, por lo que el "problema crítico» simplemente no se planteaba ${ }^{17}$. Por esto mismo, como lo han mostrado otros autores, el escepticismo no existía como una doctrina coherente y sistemática ${ }^{18}$.

Un ejemplo particularmente notable en lo que atañe al tratamiento sistemático del realismo medieval es Dominik Perler, quien ha expresado su punto de vista en términos sumamente parecidos a los de John Searle. En una obra sobre la noción de «intencionalidad» en la filosofía medieval, comparando a los autores medievales con Brentano, señala que lo propio de la epistemología en cuestión era «explicar la estrecha relación que podía existir entre el mundo material y el mundo psíquico ${ }^{19}{ }$ », siempre sobre la base de que los filósofos de tal período «estaban todos convencidos (tanto por razones metafísicas como teológicas) de que el mundo material existe y de que nuestros pensamientos están en relación con ese mundo ${ }^{20}$. Como agrega el mismo Perler en otro trabajo, lo importante para los pensadores de ese período no era «establecer que podemos obtener un conocimiento, sino explicar cómo lo adquirimos», por lo que se interesaban en dar cuenta de la «clase de procesos

16 Haldane, J., «Insight, Inference, and Intellection», en Proceedings of the American Catholic Philosophical Association 73 (1999), pp. 31-45. DOI: 10.5840/acpaproc1999733.

17 Esta opinión ha sido también desarrollada por otros autores a propósito de este período. Cf. Spruit, L., Species intelligibilis: From Perception to Knowledge. I Classical Roots and Medieval Discussions, Brill, Leiden, 1994, pp. 4-19; Thro, L. J., Is there a distinctively thomistic realism, en L'homme et son destin. D'après les penseurs du Moyen Âge, Nauwelaerts (Louvain), Béatrice-Nauwelaerts (Paris), 1960, pp. 571-576. Sobre esto se ha insistido principalmente a propósito de Tomás de Aquino. Cf. GILson, É., Le réalisme méthodique, Vrin, Paris, 1935; Gilson, É., Réalisme thomiste et critique de la connaissance, Vrin, Paris, 1939; Maritain, J., Les degrés du savoir, en Euvres Complètes. Volume IV, Éditions Universitaires/ Saint Paul, Paris-Fribourg, pp. 393-400.

18 Cf. Lagerlund, H, "A History of Skepticism in the Middle Ages», en Rethinking the History of Skepticism. The Missing Medieval Background (ed. H. Lagerlung), Brill, LeidenBoston, 2010, pp. 1-28; Perler, D., "Skepticism», en The Cambridge History of Medieval Philosophy. Volume I (Ed. Pasnau, R.; Van Dyke, C.), Cambridge University Press, Cambridge, 2010, pp. 384-385.

19 Perler, D., Théories de l'intentionnalité au Moyen Âge., Vrin, Paris, 2001, p. 30.

$20 \quad$ Ibid., p. 18. 
[que] eran requeridos para la adquisición de conocimiento ${ }^{21}$. Pues bien, estas dos formas de abordar la cuestión del conocimiento se encuentran presentes, como lo hemos visto, en Searle. Por un lado, los filósofos medievales intentaban explicar cómo adquirimos los conocimientos perceptivos que poseemos, suponiendo sistemáticamente que son conocimientos genuinos que manifiestan la realidad tal cual es. Por otro lado, la razón por la que explicar el «cómo» de la percepción es algo problemático se encuentra en el hecho de que, al igual que para Searle, la experiencia perceptiva es de naturaleza diferente a la realidad física que da a conocer, punto al que ciertos autores, Olivi por ejemplo, consagraban una atención especial ${ }^{22}$.

Podemos concluir que, tanto para los filósofos medievales como para Searle, todo el problema del conocimiento perceptivo supone que este manifiesta la realidad tal cual es, cosa que no se intenta demostrar. Como Perler lo ha puesto de manifiesto, cada vez que se abordaba el problema sobre la posibilidad del conocimiento y se examinaban los argumentos escépticos, esto se hacía con un propósito metodológico que podía servir para demostrar la superioridad de alguna teoría perceptiva con respecto a sus rivales ${ }^{23}$. Searle, por su parte, juzga que demostrar el hecho de que tenemos un acceso genuino a la realidad es algo, en estricto rigor, imposible, como también lo es el demostrar la falsedad del idealismo ${ }^{24}$. Por ello, se puede decir que, tanto para Searle como para los medievales, el realismo actúa como un presupuesto de toda la reflexión y, por esto mismo, como un principio de la argumentación. Esto es, dicho punto de partida funciona como un criterio crucial cuando se desea evaluar la capacidad que una u otra teoría posee para explicar el fenómeno del conocimiento. Así, toda argumentación asume la siguiente estructura: (1) se define el fenómeno del conocimiento sensible como una experiencia subjetiva que presenta la realidad tal cual es y donde dicha experiencia se distingue del objeto o cosa en sí conocida; (2) se elabora una teoría donde cada elemento debe explicar cómo se realiza dicho conocimiento. El realismo no es nunca aquí una hipótesis a defender, sino que la premisa fundamental. En cuanto tal, no puede prestarse a ninguna clase de demostración auténtica.

A causa de lo anterior, el escepticismo en torno a la posibilidad de tener un acceso genuino a la realidad física por medio de la percepción se descarta en el mismo punto de partida. Sobre todo para los autores medievales, el escepticismo no es ni siquiera una tesis rival que pueda prestarse para el examen. Por ello, la

21 Perler, D., «Scepticism and Metaphysics», en The Oxford Handbook of Medieval Philosophy (ed. Marenbon, J.), Oxford University Press, New York, 2012, p. 547: «Medieval philosophers committed to the Aristotelian tradition do not seem to have asked the fundamental question whether or not we can have knowledge of an external world. Their main concern was not to establish that we can have knowledge but to explain how we acquire it. They entered into lengthy discussions about following problems: What kind of cognitive processes are required for the acquisition of knowledge? What kind of knowledge do we gain by means of these processes? And what are the objects of our knowledge?».

22 Perler, D., Théories de l'intentionnalité au Moyen Âge, op.cit., pp. 50-51, 65-68; Toivanen, J., Perception and the Internal Senses. Peter of John Olivi on the Cognitive Functions of the Sensitive Soul, Brill, Leiden-Boston, 2013, pp. 120-135, 141-145.

${ }_{23}$ Perler, D., «Skepticism», en The Cambridge History of Medieval Philosophy. Volume I (Ed. Pasnau, R., Van Dyke, C.), Cambridge University Press, Cambridge, 2010, pp. 384-396.

${ }^{24}$ Seeing things, pp., 218-222. 
pregunta sobre la posibilidad del conocimiento simplemente no se planteaba, salvo algunas excepciones. Una de las más notables es, sin duda, la de Enrique de Gante, pero este acaba por rechazar el escepticismo sin siquiera intentar ofrecer argumentos realmente concluyentes en su contra. De hecho, plantea el problema con el único propósito de dar a conocer las consecuencias indeseables de una teoría del conocimiento intelectual que no incluya la iluminación divina ${ }^{25}$.

En un contexto como este, es natural que el problema crítico no fuera ni siquiera pertinente. Por su parte, John Searle se posiciona de manera muy similar ante el escepticismo. Aunque reconoce que el escepticismo no puede ser refutado seriamente, sostiene que debe ser rechazado a posteriori por sus consecuencias absurdas. ¿Qué sentido tendría hacer ciencia si, el fin y al cabo, no tenemos acceso al mundo real? Según él, cuando los filósofos modernos afirman que solo tenemos acceso al contenido de nuestra propia mente e intentan desarrollar una teoría del conocimiento a partir de esa premisa, hacen como si «[intentaran] desarrollar las matemáticas sobre la premisa de que los números no existen ${ }^{26}$. Esta actitud habría conducido a la epistemología moderna a ocuparse de una serie de temas que carecen de un interés filosófico auténtico ${ }^{27}$. Peor aún, si se sostiene que no podemos captar las cosas en sí mismas, entonces es imposible intentar tener alguna noticia sobre la existencia del mundo externo, lo cual sería desastroso para la idea que tenemos de la ciencia y de su progreso ${ }^{28}$. Por todo esto, Searle cree que el problema crítico fundado por Descartes debe abandonarse y, por ende, que la cuestión sobre la posibilidad del conocimiento no debiera tomarse demasiado en serio, aun cuando él mismo le consagre algunas páginas ${ }^{29}$.

\section{ReALismo DiREcto vS REALISMO METAFísico}

Ya reconocidas las similitudes, ahora debemos identificar algunas diferencias entre el punto de partida medieval y el punto de partida de Searle, basándonos principalmente en su última obra. La más clara reside en el hecho de que, para el filósofo norteamericano, el punto de partida descrito es incompatible con cualquier clase de «representacionalismo» (Representative Theory). Define al representacionalismo como toda teoría que intente explicar el conocimiento sirviéndose del concepto de «semejanza» (resemblance). Esto es, frente a la pregunta sobre «cómo las características específicas del campo visual ontológicamente subjetivo presentan la características del campo objetivo», los representacionalistas responden que «la representación es explicada por una relación de semejanza o de isomorfismo entre lo que representa [representing] y lo representado [represented]» ${ }^{30}$. Dicho de otra manera, que la representación esté efectivamente referida a la realidad extra-mental que está representando

25 Perler, D., «Skepticism», op.cit., pp. 385-387.

26 Seeing things, p. 29, 94.

$27 \quad$ Ibid., pp. 29, 94, 217.

28 Ibid., pp. 23-24, 94, 217-222.

29 Ibid., pp. 217-221.

30 Ibid., p. 118: "The idea, common to philosophers from Locke and Descartes right through Wittgenstein's Tractatus, is that representation is explained by a ressemblance relation or isomorphism between the representing and the represented». 
- y que lo haga con fidelidad a lo que aquella realidad es en sí- se explicaría por la semejanza existente entre las dos. Según Searle, esta teoría, «común entre los filósofos desde Locke y Descartes hasta Wittgenstein» cae en el error de concebir la representación como el objeto directo de la percepción, en lugar de reservar este título a la realidad objetiva misma a que dicha representación se refiere $^{31}$. La fuente de este error residiría en una idea dominante a partir del siglo XVII según la cual «nunca percibimos directamente objetos ni el estado de las cosas del mundo, sino que solo percibimos directamente nuestras experiencias subjetivas» ${ }^{32}$. El único argumento favorable a la tesis en cuestión sería que la experiencia subjetiva de la percepción de algo real puede ser exactamente igual a la experiencia subjetiva implicada en una alucinación (commonality of experience). Según este argumento, dado que no habría nada que permita distinguir estas dos experiencias subjetivas, se tendría que concluir que toda percepción tiene como objeto directo la representación subjetiva y no la cosa representada. Lo anterior parece implicar, además, que nunca sabemos si percibimos la realidad objetiva, puesto que siempre es posible que estemos teniendo una suerte de alucinación ${ }^{33}$. Esta última consecuencia es, según Searle, desastrosa, dado que conduciría al escepticismo y daría lugar a dificultades insolubles ${ }^{34}$. Ahora bien, Searle sostiene que el anterior argumento - que califica como el "mal argumento" (the bad argument) - es inválido, por estar fundado en una falacia de equivocidad. Basándose en una distinción que ya existía en Intentionality ${ }^{35}$, Searle sostiene que este argumento confundiría dos cosas diferentes que tienen lugar dentro de todo acto perceptivo: (1) nuestra experiencia subjetiva que consiste en percibir una cosa; (2) nuestra experiencia subjetiva de la percepción misma ${ }^{36}$. Pues bien, en el caso de la experiencia verídica, efectivamente se puede decir que "percibimos tal o cual objeto». No obstante, en el caso de la alucinación, «no estamos percibiendo nada» y solo tenemos una experiencia sin objeto. En el primer caso, tenemos un estado mental «de» algo diferente al estado mental mismo; en el segundo caso, en cambio, tenemos un estado mental «del» estado mental mismo. En consecuencia, aunque las dos sean fenomenológicamente idénticas, no se puede inferir que, en los dos casos, lo que se percibe directamente sea la experiencia subjetiva ${ }^{37}$.

El anterior argumento permite aclarar un punto importante: el «percibir» mismo de una realidad no puede percibirse. Lo perceptible es la realidad captada por ese percibir. De ello se sigue que toda buena definición de la percepción debe admitir que su objeto directo es la realidad extra-mental y no la percepción misma ${ }^{38}$. Con esto, Searle pretende redefinir los términos básicos de todo debate gnoseológico con el propósito de desligarlo de raíz del problema crítico.

\footnotetext{
31 Ibid., p. 119.

32 Ibid., p. 11.

33 Ibid., pp. 20-24.

$34 \quad$ Ibid., p. 23.

35 Cf. Intentionality, pp. 2; 37-40.

36 Seeing things, pp. 24-25.

37 Ibid., pp. 24-29.

38 Ibid.. pp. 27, 76, 107, 217. Por ejemplo: «One thing you cannot see when you see anithing is your seeing of that thing» (p. 107). Cf. Intentionality, p. 38.
} 
No me pronunciaré aquí sobre la pertinencia del argumento de Searle, ya que habría que examinarlo con más detalle y su autor le consagra muchas páginas ${ }^{39}$. Lo que debemos retener aquí es la tesis que el filósofo norteamericano estima como la más apropiada: toda percepción verídica tiene como objeto directo la cosa percibida, no el acto perceptivo. Ahora bien, es precisamente en este punto donde la posición de Searle revela una particularidad bastante extraña para cualquier historiador de la gnoseología medieval. El autor parece creer que afirmar que «toda percepción verídica tiene como objeto directo la cosa percibida» equivale a afirmar que «toda percepción verídica incluye únicamente el objeto y la experiencia perceptiva, excluyéndose cualquier entidad intermedia que la mente emplee como medio por el cual se percibe el objeto». Esta última afirmación se identifica con lo que el mismo Searle denomina "realismo directo» (Direct Realism). En su propias palabras, la anterior teoría incluye la idea de que no necesitamos percibir, primero, algo diferente de la cosa por lo cual (by way of which) se acceda a un conocimiento perceptivo de ella $^{40}$. Bastan la experiencia subjetiva y la realidad objetiva que es percibida gracias a la primera. El no reconocimiento de lo anterior conduciría necesariamente al «representacionalismo» tal como Searle lo define: lo percibido directamente es la experiencia subjetiva y no la realidad en sí misma. Y, más aún, el rechazo de dicho «realismo directo» traería consigo todos los desastres epistemológicos implicados en las teorías del conocimiento desarrolladas desde Descartes ${ }^{41}$.

Ahora bien, para cualquiera que conozca la epistemología medieval de los siglos XIII y XIV, es fácil ver que Searle confunde dos cosas muy diferentes a causa de cierta ambigüedad implicada en la expresión «realismo directo». En efecto, esta expresión puede referirse a dos cosas: (1) una definición del conocimiento que se presenta como un punto de partida de todo debate posible sobre él y que, por ende, se revela indemostrable; (2) una posible teoría explicativa del conocimiento que, en cuanto tal, exige razones adicionales que la apoyen. Así, no es lo mismo admitir que efectivamente podemos acceder directamente a la realidad extra-mental y conocerla tal cual es en sí misma que afirmar que lo hacemos sin necesidad de ninguna entidad intermedia tal como una «semejanza» o una «especie». Lo primero se identifica con el realismo tal como fue descrito en la anterior sección y forma parte de aquellos puntos de vista que, por ejemplo, Popper juzgaba «indemostrables» por no prestarse a ninguna demostración ${ }^{42}$; lo segundo, en cambio, es una tesis gnoseológica que intenta explicar el fenómeno del conocimiento bajo el supuesto de que se acepta la anterior definición, exigiendo, por ende, argumentos que prueben su superioridad respecto de otras teorías rivales que operan bajo el mismo supuesto.

La anterior distinción estaba perfectamente clara para los filósofos medievales. Estos creían, en su gran mayoría, que «aquello que es percibido» no es la experiencia

39 Seeing things, pp. 10-32, 80-99.

40 Ibid., p. 17: "When I say the objective state of affairs is directly perceived, I mean you do not have to first perceive something else by way of which, or by means of which, it is perceived. Your experience, to repeat, is not like seeing something on television in a mirror».

41 Ibid, p. 19: "I said the denial of Direct Realism was disastrous and I want now briefly to say how» (p. 19). Cf. Ibid., pp. 29-32, 107, 94-99, 217-218.

42 Popper, K, Objective Knowledge. An Evolutionary Approach, Oxford University Press, Oxford, 1979, pp. 38-44. 
subjetiva, sino que la cosa misma ${ }^{43}$. En este punto, habrían estado de acuerdo con Searle y, probablemente, habrían dado su apoyo a su argumento de que «el mal argumento" se funda en una falacia de equivocidad que suscitaría una manera incorrecta de cuestionarse sobre la esencia del fenómeno del conocimiento. Sin embargo, ocurre que la adopción del modo mencionado de abordar el fenómeno en cuestión no era equivalente a la defensa de una posición similar al realismo directo propuesto por Searle. Creer que «lo percibido» era la cosa y no «el acto perceptivo» o "cualquier cosa implicada en el acto perceptivo» no equivalía necesariamente a creer que la percepción no incluía ninguna entidad «por medio de la cual» o «en la cual» se accediera a la realidad. Ciertamente, hubo autores tales como Olivi y Ockham que defendieron un «realismo directo» como el de Searle ${ }^{44}$. No obstante, no situaban en él la condición de posibilidad para adoptar la idea de que "lo percibido» es la cosa misma y no «la experiencia subjetiva». Hacían, en verdad, exactamente lo contrario: cuando defendían un punto de vista como el de Searle, se apoyaban en la forma mencionada de definir la percepción y el conocimiento, asumiendo esto último como una premisa que se distinguía claramente de la teoría misma. Por ejemplo, Pedro de Olivi, filósofo de la segunda mitad del siglo XIII, sostenía que nuestra percepción de la realidad no necesita, como lo creían otros autores antes que él, de ninguna entidad intermedia por la cual dicha realidad se percibiera, porque, entre otras razones, estimaba que un esquema explicativo de esa índole implicaría que lo conocido no sería la realidad misma, sino que la entidad intermedia por la cual se accede a ella (entidad que los medievales llamaban «especie») ${ }^{45}$. De este modo, para el filósofo franciscano, por un lado, estaba la definición correcta de la percepción, según la cual esta se encontraría directamente referida a una realidad extra-mental; por otro lado, estaba la tesis que elimina las entidades intermedias en la explicación de la percepción y del conocimiento. Para Searle, en cambio, estas dos cosas se identifican. Su "realismo directo», que posee características similares al «realismo directo» de Olivi ${ }^{46}$, es simplemente lo mismo que la buena definición

43 Toivanen, J., Perception and the Internal Senses, op.cit., p. 124: "It is important to note that Aristotelian species theories do not intend to introduce sensible species as kinds of representational entities which would be the immediate objects of cognitive acts. Rather, they are causal intermediaries which account for the causal relation between an external object and a cognitive power, and the object that is perceived is the external object itself. In this way, these theories at least pretend to avoid representationalism and adhere to direct realism». Cf. Spruit, L., Species intelligibilis, op.cit., p. 7, 10-12.

44 Pasnau, R., Theories of Cognition in the later Middle Ages, Cambridge, Cambridge University Press, 1997, pp. 161-194, 220-290; Perler, D., «Essentialism and Direct Realism. Some Late Medieval Perspectives», en Topoi 19 (2000), pp. 116-119. DOI: 10.1023/A:1006425008627; Perler, D., Théories de l'intentionnalité au Moyen Âge, op.cit., pp. 43-75; Spruit, L., Species intelligibilis, op.cit., pp. 215-223; 228-298; Toivanen, T., Perception and the Internal Senses, op.cit., pp. 120-139.

45 Para un desarrollo sistemático de este argumento, cf. Perler, D., Théories de l'intentionnalité au Moyen Âge, op.cit., pp. 51-60; Toivanen, J., Perception and the Internal Senses, op.cit., pp. 120-139.

46 Esta similitud se puede comprobar comparando el esquema propuesto por Alain de Libera para caracterizar la teoría del conocimiento de autores como Olivi con los esquemas que utiliza Searle para caracterizar su propia teoría. Cf. DE LiBERA, A., Archéologie du sujet III. La double révolution, Paris, Vrin, 2014, p. 144; Seeing things, pp. 18-19; Intentionality, pp. 57-59. 
de la percepción y del conocimiento. Así, lo que para Olivi se presentaba como una premisa y, por ende, como un argumento a favor de su propia teoría explicativa del conocimiento, para Searle se identifica con la teoría.

Probablemente, la confusión de Searle se explica por el hecho de que su definición del representacionalismo es demasiado amplia, incluyendo dentro de él teorías que no se oponen necesariamente a la definición correcta de la percepción. De acuerdo a esta definición, el representacionalismo incluiría toda teoría que afirme la necesidad de entidades intermedias de orden subjetivo para percibir la realidad. Según él, el error de esta clase de teorías residiría en afirmar que el objeto de nuestro conocimiento perceptivo se identifica con dicha entidad intermedia o «representación», la cual mantendría una relación de semejanza con la cosa a la que se refiere. En este punto, tiene razón en pensar que esta manera de concebir la percepción es errada, ya que simplemente se opone a lo que la percepción es por definición: lo percibido es siempre una cosa independiente de la percepción ya que, de lo contrario, no hay realmente una percepción. Más aún, es absurdo pensar que el acto mismo de percepción sea percibido, puesto que este es, por principio, imperceptible, tal como se indicó anteriormente. No obstante, Searle se equivoca al pensar que toda teoría de la percepción que afirme la necesidad de que existan entidades intermedias sea opuesta a la anterior definición de la percepción. Es perfectamente concebible que haya una teoría explicativa de la percepción que admita la necesidad de dichas entidades, al mismo tiempo que adopte como punto de partida de su análisis la definición en cuestión. En efecto, es teóricamente coherente pensar que, por un lado, la percepción tiene la realidad extra-mental como su objeto directo y, por otro lado, que este sea alcanzado por medio de una entidad intermedia "ontológicamente subjetiva». Como se señaló, casi todos los filósofos medievales defendieron teorías de la percepción con la anterior estructura. Un ejemplo particularmente notable lo encontramos en Tomás de Aquino. En diversas ocasiones, este afirma que lo que se conoce en todo acto de conocimiento es la cosa misma. Es una cosa independiente del sujeto cognoscente a lo que todo acto cognitivo está referido. Sin embargo, al mismo tiempo, Tomás afirma varias veces la tesis de que este acceso directo a la realidad se lleva a cabo "por medio» de o «en» las «especies» (species) que recibe el intelecto de dicha realidad ${ }^{47}$. Esta última clase de declaraciones incluso ha conducido a que ciertos historiadores de la filosofía medieval califiquen la teoría tomasiana de "representacionalista» ${ }^{48}$, lo que, a mi juicio, ha sido debidamente refutado ${ }^{49}$. En efecto, el Aquinate declara, en numerosas ocasiones, que todo conocimiento, a pesar de necesitar las «especies», tiene a la realidad misma como objeto directo. Las especies no son nunca el objeto conocido ya que, de serlo, serían las especies mismas lo que se conoce y no la

\footnotetext{
47 Cf. Tomás de Aquino, Suma teológica, I, q. 85, a. 2, c.; Comentario al libro Del alma, III, 2 (Leonina XLV-1, p. 213).

48 Pasnau, R., Theories of Cognition in the later Middle Ages, op.cit., pp. 195-220; Panaccio, C., Aquinas on Intellectual Representation, in Ancient and Medieval Theories of Intentionality, Brill, Leiden-Boston-Köln, 2001, pp. 185-202.

49 Macdonald, P. A., «Direct Realism and Aquinas's Account of Sensory Cognition», en The Thomist 71, 3 (2007), pp. 343-378; Perler, D., "Essentialism and Direct Realism», op.cit.,
} pp. 111-116. 
realidad a la cual dichas especies se refieren. Por ende, estas especies no son más que aquello "por medio de lo cual» es conocida la realidad a la cual están apuntan.

Como se puede observar, la tesis de que «la realidad extra-mental es lo que se conoce directamente en la percepción» no se opone lógicamente a la idea de que «la realidad extra-mental se conoce por medio de una entidad mental». Podrá, ciertamente, haber argumentos que conduzcan a rechazar teorías como esta última pero, lógicamente hablando, no se contrapone de manera obvia a la primera. Prueba de ello es que se puede elaborar toda una teoría para justificar dicha compatibilidad, teoría que parece estar presente, por ejemplo, en los escritos de Tomás de Aquino. Según este filósofo, toda la realidad física es una composición esencial de "materia» y "forma», donde la forma es de orden inmaterial y posee toda la información relativa a la esencia de una cosa. Pues bien, todo acto de conocimiento consiste precisamente en la presencia real de la forma de una cosa en el sujeto que la conoce. En consecuencia, cuando se dice que la realidad es conocida por medio de la especie, lo que se está diciendo es que la forma misma de la realidad se hace realmente presente en la mente, si bien de modo inmaterial. Esto responde a la pregunta de cómo se puede decir que se conoce la realidad concreta cuando esta es captada por medio de una entidad que no se identifica con ella. La respuesta es que la especie y la realidad sí se identifican, puesto que la forma conocida y la forma que constituye a la cosa son una y la misma cosa asumiendo dos modos diversos de existencia ${ }^{50}$.

Searle podría, ciertamente, tener buenos argumentos para rechazar la anterior teoría. No obstante, esto no neutraliza la objeción que se está examinando aquí por el simple hecho de que no se está intentando demostrar que el punto de vista de Searle sea falso. Lo que se está tratando de probar es que no es contradictorio reconocer que la percepción tiene la realidad extra-mental como objeto directo y admitir, a la vez, que esa realidad se capta por medio de una entidad mental. La teoría tomista que acaba de ser descrita podrá ser, tal vez, falsa, pero da cuenta de la posibilidad de concebir el conocimiento perceptivo de manera que estos dos elementos cohabiten sin que haya contradicción. Por consiguiente, no basta con definir la percepción en los términos de Searle para concluir que solo el realismo directo defendido por él es admisible. De ahí que su argumentación se revele, al fin y al cabo, insuficiente. El filósofo norteamericano hubiese evitado esta insuficiencia de haber conocido los debates actuales sobre la epistemología medieval. Ciertamente, ello hubiese contribuido positivamente en su teoría cuya argumentación hubiera sido, sin duda, más rica.

Más aún, me parece que el modo de argumentar de Searle es circular. En efecto, como se mencionó anteriormente, toda la refutación del representacionalismo reposa sobre lo que él juzga como una buena definición del conocimiento perceptivo. Esta buena definición inicial permitiría que el fenómeno de la percepción se revele como claramente diferente de la experiencia subjetiva que ha lugar en

50 Cf. Klima, G., «Tradition and Innovation in Medieval Theories of Mental Representation», en Proceedings of the Society for Medieval Logic and Metaphysics 4 (2004), pp. 4-11. <http://www.fordham.edu/gsas/phil/klima/SMLM/PSMLM4/PSMLM4.pdf>; MACDONALD, P. A., «Direct Realism and Aquinas's Account of Sensory Cognition», op.cit., pp. 343-378; PerLer, D., «Essentialism and Direct Realism», op.cit., pp. 111-116. 
una alucinación o algo por el estilo, a pesar de que sean fenomenológicamente idénticas. Luego, concluye que su versión del «realismo directo» es la única teoría del conocimiento perceptivo apta para no caer en esta confusión, puesto que la buena definición de este conocimiento simplemente se identificaría con la teoría en cuestión. Pero esto, ¿no es acaso suponer la verdad de su teoría desde el comienzo? En efecto, Searle piensa que la explicación de la intencionalidad del conocimiento perceptivo presupone una correcta definición de este para evitar la ambigüedad descrita. Según esta buena definición, el objeto directo de toda percepción es la realidad extra-mental a la cual dicha percepción se refiere. Hasta aquí, no hay problemas. No obstante, lo que no ve Searle es que lo único que ha hecho aquí es definir claramente el punto de partida de toda investigación que pretenda explicar la naturaleza del conocimiento perceptivo, por lo que no ha dicho nada aún sobre la necesidad de negar una u otra de las teorías rivales, ni mucho menos de afirmar una de ellas. Esto exige, en conformidad con lo expresado más arriba, argumentos adicionales. En lugar de esto, Searle se limita a reafirmar la definición en cuestión, como si eso equivaliera a probar la verdad del realismo directo como teoría. En resumen, Searle define la percepción en términos que, en rigor, no dan necesariamente la razón a su realismo directo, dando por hecho, no obstante, y desde el inicio, que esos términos incluyen lo que solo pertenece a su teoría: que la explicación de un acto perceptivo está suficientemente descrita sin recurrir a entidades subjetivas como «semejanzas» o «especies». Como esto último es justamente lo que está en discusión — tal como se puede observar en los debates medievales - se puede decir que Searle asume la verdad de su conclusión como una premisa incluida implícitamente en su definición del acto perceptivo.

\section{LA CUESTIÓN DEL ESCEPTICISMO}

De manera similar, el rechazo sistemático a la cuestión de la posibilidad del conocimiento presenta ciertas diferencias entre Searle y los filósofos medievales. En efecto, Searle parece creer que el representacionalismo condiciona, de suyo, el que los temas tratados en torno al conocimiento sean limitados a resolver la cuestión sobre la posibilidad de conocer la realidad, cuestión que se puede formular en estos términos: «(...) dado que todo lo que podemos percibir son nuestros propios datos sensibles [sense data], ¿cómo es posible obtener un conocimiento seguro y cierto del mundo externo? ${ }^{51}$. Como piensa que este modo de abordar el tema de la percepción y del conocimiento en general conduce al escepticismo, concluye que esta clase de cuestionamientos debe ser abandonada. Ahora bien, como, según él, tales cuestionamientos dependen directamente de la tesis representacionalista, entonces esta tesis también debe ser rechazada.

Pues bien, ocurre que los pensadores medievales no necesitaban oponerse al «representacionalismo» en ninguna de sus formas para creer que la cuestión sobre la posibilidad del conocimiento no merece un lugar privilegiado dentro de

51 Seeing things, p. 217: «Specifically, given that all we can perceive are our own sense data, how is it possible to gain secure and certain knowledge of the external world?». 
la gnoseología. Más aún, como ya fue mencionado, la mayoría de estos autores proponían teorías de la percepción que, de acuerdo a la definición amplia de Searle, podrían ser calificadas de «representacionalistas», porque sostenían, siguiendo a Aristóteles, que la realidad se conoce por medio de «especies» o de "semejanzas». Pero lo importante aquí es que aquella cuestión ni siquiera habría jugado un rol crucial en un autor que hubiese adoptado alguna forma de representacionalismo en un sentido más estricto que el definido por Searle, como de hecho los hubo ${ }^{52}$. El rechazo de esta clase de preguntas era, para ellos, anterior a la defensa de una u otra teoría del conocimiento, aun cuando, en ciertas ocasiones, estimaran útil servirse de las razones escépticas que conocían por los antiguos para reducir al absurdo alguna teoría rival. Por consiguiente, la adopción de una u otra teoría explicativa de la percepción daba por sentado que el escepticismo no era una opción y, por ende, ninguna de dichas teorías se podía prestar para suscitar el tipo de preguntas que, según Searle, son una consecuencia del representacionalismo. Se partía de la base de que podemos conocer la realidad tal cual es y, si ciertas razones los conducían a adoptar una teoría explicativa abiertamente representacionalista, simplemente no creían que fuera necesario rechazar ese punto de partida. Con menor razón pensaban que hubiese que defender una teoría como la de Searle — que niega toda entidad intermedia entre la percepción y la realidad- para adoptarlo. Y la razón es muy simple: aceptar la idea de que podemos conocer la realidad perceptivamente no era una consecuencia que proviniese de alguna teoría del conocimiento, sino que, por el contrario, era una idea que solo podía ser el resultado de una concepción metafísica más amplia ${ }^{53}$.

El que algunos pensadores como Olivi y Ockham hayan rechazado la teoría de las especies por juzgar que estas últimas «velarían» la realidad más que darla a conocer no es prueba de lo contrario. A diferencia de Searle, no pensaban que la aceptación de las especies condicionara la reflexión filosófica en el sentido de limitarla a la cuestión por la posibilidad del conocimiento. Muy por el contrario, creían que si el objetivo era explicar el fenómeno del conocimiento, entonces este no quedaba realmente explicado por medio del concepto de especie por la razón que acaba de ser mencionada.

Una vez más, por lo tanto, se puede ver que todo el problema de Searle reside en el hecho de que no es capaz de distinguir, por un lado, el punto de partida que admite que la percepción es, por definición, la captación directa de una realidad extramental y, por otro lado, la tesis que defiende la idea de que todo acto perceptivo se explica suficientemente sin recurrir a ningún tipo de entidad intermedia subjetiva como lo son las especies. Esta confusión lo conduce, en su rechazo del problema crítico y del escepticismo, a una argumentación circular similar a la mencionada en la sección anterior. Su rechazo al escepticismo lo empuja a defender su propia versión del realismo directo, pero, al mismo tiempo, hace depender de ella la

52 Cf. Pasnau, R., Theories of Cognition in the later Middle Ages, op.cit., pp. 89-100. Aunque es cierto que la tendencia a ocuparse del tema del conocimiento desde una perspectiva representacionalista parece haber tenido como resultado el que la pregunta por la posibilidad de conocer fue asumiendo, poco a poco, un papel más preponderante. Cf. Spruit, L., Species intelligibilis. From Perception to Knowledge. Volume II, Brill, Leiden-New York-Köln, 1995.

53 Perler, D., «Scepticism and Metaphysics», op.cit., pp. 547-565. 
falsedad del escepticismo. Un conocimiento de los trabajos de los historiadores de la filosofía medieval le hubiese permitido evitar esta consecuencia negativa, permitiéndole plantear las cosas en los siguientes términos: (1) aceptación de la posibilidad de percibir la realidad tal cual es; (2) rechazo del problema crítico y del escepticismo; (3) definición de la percepción según la cual su objeto directo es la realidad extra-mental y no la experiencia perceptiva misma; (4) argumentación a favor de su rechazo de la necesidad de entidades intermedias que posibiliten la captación de la realidad. Los puntos número (1), (2) y (3) forman parte del punto de partida. Solo el punto (4) se presta para la discusión y para el debate, por lo que no es correcto hacer depender de él los tres primeros, básicamente porque afirma una idea que no puede ser ni siquiera defendida sin presuponerlos.

\section{4. ¿ES POSIBLE PRESCINDIR DE LAS «ESPECIES»?}

La teoría de Searle presenta una serie de particularidades que la vuelven, sin ninguna duda, extremadamente interesante. Más aún, debe admitirse que la adopción de un punto de partida realista le permite desarrollar una teoría de la percepción con un nivel de detalle que difícilmente puede darse cuando se reconoce legitimidad al problema crítico. Ahora bien, en relación con la gnoseología medieval, conviene examinar un aspecto esencial de esta teoría. Recordemos que Searle estima que la pregunta principal a responder en toda teoría de la percepción consiste en explicar cómo un fenómeno ontológicamente subjetivo puede presentar tal cual es una realidad ontológicamente objetiva, lo cual se identifica, según él, con el problema de la intencionalidad del conocimiento. Dicho de otro modo, ¿cómo una realidad esencialmente diferente a otra es capaz no solo de referirse a ella sino que de «presentarla» fielmente? El aspecto que será analizado es la respuesta dada por Searle a esta pregunta.

Recuérdese que lo esencial de su posición es que intenta explicar la percepción afirmando la verdad de dos ideas: (1) el objeto directo de la percepción es la realidad extra-mental; (2) esta no necesita ninguna entidad por medio de la cual este objeto sea captado. Ya nos hemos ocupado de la primera idea y hemos visto que, erróneamente, Searle tiende a confundirla con la segunda. Lo que haremos ahora es explicar qué tipo de teoría ofrece Searle para explicar esta última. En otras palabras, ¿cómo explica la percepción prescindiendo por completo de recurso de la «especie» o de la «semejanza»?

Searle plantea que las cosas materiales pueden causar la percepción por el hecho de que poseen la capacidad de provocar el tipo de percepción que provocan:

«La hipótesis que quiero explorar es que la explicación de cómo las características cualitativas (qualitative features) de la experiencia visual presentan, en el caso de las características básicas (basic features), las condiciones de satisfacción que presentan, es que hay una relación sistemática entre la propiedad de ser ' $F$ ' y la propiedad de ser capaz de causar una cierta clase de experiencia ${ }^{54}$.

\footnotetext{
$54 \quad$ Seeing things, p. 121.
} 
Como se puede observar, Searle propone que aquello que vuelve posible que un acto de percepción presente una realidad tal cual es en sí misma radica en el hecho de que dicha realidad es, por naturaleza, apta para causar aquella clase de acto de percepción. De este modo, el contenido específico de cada acto de percepción se debe a que la realidad a la cual se refiere posee un modo de ser que incluye la capacidad de provocarlo tal cual se presenta. En el texto citado, Searle se está refiriendo únicamente a un tipo específico de experiencia visual, a saber, a aquel que este autor denomina como «características básicas» (basic features), pero pretende extender esta explicación a todo tipo de acto perceptivo ${ }^{55}$.

¿Qué podemos decir de esta tentativa por explicar la percepción sin recurrir a las «especies» o «similitudes»? Recordemos que Searle está intentando defender la idea de que no se necesita ninguna entidad intermedia entre el acto perceptivo mismo y la cosa percibida. Ahora bien, frente a esto, surge la pregunta: ¿qué es eso que posee la cosa en virtud de lo cual es susceptible de provocar el tipo de percepción que provoca en un sujeto? Me parece que todo el problema radica justamente allí. ¿Por qué el rojo tiene la capacidad de provocar la percepción visual del rojo? Tomás de Aquino, por ejemplo, diría: porque posee la «forma de rojo», es decir, en su esencia posee un elemento irreductible a lo corpóreo que determina su ser rojo. Asimismo, esa forma se traslada al ojo, posibilitando la percepción visual del rojo. Pues bien, esto es posible mediante lo que él, junto con otros, denomina «especie». Había varias teorías que buscaban explicar cómo se produce el traslado de la especie desde la realidad hasta el sujeto cognoscente, pero que no nos interesan aquí. Lo que debemos retener por el momento es únicamente la idea de que la especie es recibida por un cognoscente quien, a partir de ella, conoce la realidad. Esa especie o "intentio» permite que la cosa sea captada en su ser real, pero únicamente desde la perspectiva de su forma. En lenguaje actual, siguiendo una sugerencia de Sanguineti ${ }^{56}$, podríamos traducir el concepto de especie por «información» o como entidad que posee y traslada una cierta información de una realidad a otra de modo que pueda existir en una y otra a la vez ${ }^{57}$. Volviendo a Searle, ¿en virtud de qué cosa un proceso físico no intencional —es decir, un conjunto de procesos materiales que ocurren en la realidad extra-mental y en los órganos sensoriales- se presta para dar lugar a un contenido perceptivo de orden cualitativo e intencional? Hablar de «aptitud para provocar tal o cual tipo de percepción» supone justamente la presencia de una «información» en los dos extremos del proceso, es decir, de algo presente tanto en el objeto conocido como en la percepción. Por lo tanto, es legítimo pedirle a esta teoría que explique qué es aquello que sustenta esa información en uno u otro extremo. Muchos filósofos medievales habrían respondido a esa pregunta recurriendo a las nociones de «forma» y de «especie». El problema de Searle, por lo tanto, no es que su propuesta

55 Para el desarrollo detallado de la teoría, cf. Seeing things, pp. 121-134.

56 Sanguineti, J. J., «La especie cognitiva en Tomás de Aquino», en Tópicos 40 (2011), p. 70 .

57 Podría objetarse: ¿qué autoriza a afirmar que esa especie garantiza que la forma de la misma cosa percibida se haga presente en el sujeto que la conoce? Ya algunos historiadores se han ocupado de esta cuestión. Por ejemplo, Klima, G, «Tradition and Innovation in Medieval Theories of Mental Representation», op.cit., pp. 4-11. 
sea incorrecta, sino que es incompleta. Frente a la doble pregunta, ¿cómo un mismo contenido específico puede, por un lado, encontrarse en un sujeto y en un objeto y, por otro lado, trasladarse del primero al segundo?, no da una respuesta completamente satisfactoria puesto que no explica cómo el modo de ser de la cosa es susceptible de provocar la clase específica de contenido perceptivo que produce. Como se ha indicado, algunos filósofos medievales propusieron teorías de la percepción sin recurrir a las especies. Dos ejemplos notables son Olivi y Ockham. Si bien sus teorías plantean otras dificultades, desarrollan argumentos interesantes para resolver los problemas que surgen cuando se rechazan las especies, sobre todo Olivi ${ }^{58}$. Por lo tanto, es muy probable que la teoría de Searle se hubiese enriquecido argumentativamente de haberlos conocido.

En conformidad con lo anterior, se vuelve pertinente aplicar a Searle el mismo diagnóstico que Alain de Libéra hace de Thomas Reid, filósofo escocés del siglo XVIII:

«El escenario de Reid no tiene más que un defecto mayor: ignora la Edad Media. Critica las ideas en tanto que continúan a las especies, abriendo así una 'crisis de las ideas'. Pero no ve que estos escolásticos tan denigrados fueron ellos mismos los primeros en criticar las especies, abriendo así la 'crisis de las especies' para la cual la crisis de las ideas es heredera. Para decir las cosas claramente, Reid continúa un gesto medieval: hace para su propio siglo lo que Olivi, Guillermo de Ockham y tantos otros hicieron por el suyo, en los siglos XIII y XIV » ${ }^{59}$.

El defecto de Searle no consistiría únicamente en desconocer los planteamientos gnoseológicos de la Edad Media, sino en que, a causa de ello, se priva de enriquecer el suyo. En efecto, al proponer una teoría muy similar a la adoptada por filósofos tales como Olivi, pudo haberse beneficiado de algunos de sus elementos explicativos, tal como han sido desarrollados por comentadores recientes.

\section{CONCLUSIÓN}

Otro historiador importante de la filosofía medieval, Olivier Boulnois, ha dicho a propósito de la importancia del estudio de la filosofía medieval y justamente para desmarcarse del proyecto de algunos filósofos de tradición analítica, lo siguiente:

«(...) si creo necesario el estudio de la filosofía medieval es porque hay ingenuidades que soy incapaz de soportar. Cuando uno cree estar renovando la filosofía sin meditar su historia, se corre el riesgo de tartamudear las aporías del pasado. No se puede revolucionar un arte sino frecuentando a sus grandes maestros ${ }^{60}$.

Creo que, en cierto modo, Searle cae en esto. No solamente ignora por completo autores que, ocho siglos antes que él, escribieron sobre los mismos temas que le interesan y profesaron puntos de vista extremadamente parecidos a los suyos;

58 ToIvanen, J., Perception and the Internal Senses, op.cit., pp. 120-135, 145-161, 179-191

59 De LiBera, A., Archéologie du sujet III, op.cit, p. 161.

60 Boulnois, O., Métaphysiques rebelles. Genèses et structures d'une science au Moyen Âge, PUF, Paris, 2013, p. 17. 
además, deja simplemente de lado las dificultades que dichos puntos de vista suscitan. Searle no puede alegar a su favor el que esos autores sean desconocidos, ya que muchos historiadores de la filosofía vienen escribiendo hace tiempo sobre ello.

El resultado de su ignorancia no es menor. Al proponer una teoría de la percepción que revela un parecido notable con la de filósofos medievales tales como Pedro de Olivi y, más aún, al defenderla por medio de argumentos similares, se expone a sus mismas dificultades filosóficas. Por ende, la no consideración, por parte de Searle, de los finos análisis realizados por los historiadores de la filosofía medieval lo ha privado de argumentos genuinamente filosóficos y no únicamente de un saber limitado al ámbito de la historia de las ideas. Conociendo esos trabajos, es muy probable que hubiese incluido nuevos elementos teóricos que habrían enriquecido su doctrina, ya sea en lo que respecta al contenido de su tesis, ya sea por el hecho de que hubiese desarrollado argumentos capaces de neutralizar las posibles objeciones. Más concretamente, Searle hubiese estado obligado a ofrecer argumentos que dieran solución, en primer lugar, al problema de que su realismo directo no explica suficientemente cómo se vuelve posible que una misma información esté en dos lugares diferentes. En segundo lugar, Searle hubiese evitado la identificación entre el «realismo directo» como teoría y el «realismo» como punto de partida. Se puede ser realista en el segundo sentido y no serlo en el primero, sin que esto implique caer en el escepticismo. Esto último es particularmente importante, puesto que no necesitamos adoptar ninguna teoría del conocimiento específica, ni para evitar el escepticismo, ni para garantizar un punto de partida que abra la posibilidad de que nos ocupemos de las cuestiones epistemológicas que realmente interesan. Para ello, basta simplemente con adoptar un punto de partida realista apoyándonos, como lo propone Perler, en razones metafísicas.

Sirviéndonos nuevamente de las palabras de Olivier Boulnois, se puede afirmar que Searle cae en la «ingenuidad» de «tartamudear las aporías de la pasado», lo que pudo evitar de haberse interesado en la investigación filosófico-histórica. Lo mismo podría decirse de muchos otros filósofos contemporáneos, razón por la cual estimo que las conclusiones presentadas en este trabajo son válidas más allá de los límites de la obra comentada de Searle. Para resumir: el trabajo histórico-filosófico no solo contribuye a nuestro conocimiento del pasado, sino que además es susceptible de enriquecer reflexiones estrictamente filosóficas.

Universidad Adolfo Ibáñez, Chile

Facultad de Artes Liberales

José Antonio Valdivia Fuenzalida

Departamento de Filosofía

jose.antonio.valdivia.f@gmail.com

[Artículo aprobado para publicación en diciembre de 2016] 